\title{
The Impulsive Motion of Flat Plate in Generalized Second Grade Fluid with Anomalous Diffusion
}

\author{
Mohammad Tanzil Hasan ${ }^{1, ~ *, ~ M d . ~ S h a f i q u l ~ I s l a m ², ~ M i r ~ S h a r i f u l ~ I s l a m ~}{ }^{3}$ \\ ${ }^{1}$ Department of Mathematics, Bangabandhu Sheikh Mujibur Rahman Maritime University, Dhaka, Bangladesh \\ ${ }^{2}$ Department of Applied Mathematics, Dhaka University, Dhaka, Bangladesh \\ ${ }^{3}$ Department of Oceanography, Dhaka University, Dhaka, Bangladesh \\ Email address: \\ tanzil_du@yahoo.com (M. T. Hasan),mdshafiqul@du.ac.bd(Md. S. Islam), mirsislam77@gmail.com (M. S. Islam) \\ ${ }^{*}$ Corresponding author
}

To cite this article:

Mohammad Tanzil Hasan, Md. Shafiqul Islam, Mir Shariful Islam. The Impulsive Motion of Flat Plate in Generalized Second Grade Fluid with Anomalous Diffusion. American Journal of Applied Mathematics. Vol. 8, No. 6, 2020, pp. 327-333. doi: 10.11648/j.ajam.20200806.15

Received: September 26, 2020; Accepted: November 3, 2020; Published: December 11, 2020

\begin{abstract}
The flow adjacent to a wall rapidly set in motion for a generalized second-grade fluid with anomalous diffusion is examined. For the elucidation of such a fluid, the fractional-order derivative approach in the constitutive relationship model is presented because models based on ordinary differential equations have a relatively limited class of solutions, which does not provide compatible description of the complex systems in general. The current model of second-order fluid involving fractional calculus is based on the formal replacement of the first-order derivative in ordinary rheological constitutive equation by fractional derivative of a non-integer order. In addition, the time-fractional equation considered in this article describes the anomalous sub-diffusion. In this article, the velocity and stress field of generalized second-grade fluid with fractional anomalous diffusion are studied by fractional partial differential equations. Analytic solutions are given in closed form, from these differential equations in terms of the generalized G-functions or Fox's $H$-function with the discrete Laplace transform technique. Thus, many previous and classical results, namely, the solution of fractional diffusion equation obtained by Wyss, the classical Rayleigh's time-space regularity solution, the relationship between velocity field and stress field obtained by Bagley and Torvik, are represented by particular cases of our proposed derivation.
\end{abstract}

Keywords: Time Fractional Navier-Stokes Equation, Generalized Second Grade Fluid, Anomalous Diffusion, Fox's $H$-function

\section{Introduction}

In the past few decades, non-Newtonian fluids have gained increasing popularity, mainly due to their concrete application in various fields such as material processing, chemical and nuclear industries, geophysics, oil reserving engineering and bioengineering [1]. Ketchup, blood, paints, shampoo, polymer melts, oils, drilling mud, and greases are examples of non-Newtonian fluids [2, 3]. The large variety of fluids and industrial applications has been a major motivation for research in non-Newtonian flow. One of the important class of non-Newtonian fluids is viscoelastic fluid. However, even the most commonly used simplest subclass of viscoelastic fluids is the so-called second-order fluid.

Recently fractional calculus has encountered much success in the description of complex dynamics. It has proved to be a valuable tool to handle viscoelastic behavior. The starting point of the fractional derivative model of viscoelastic fluid is generally a classical differential equation, which is adjusted by substituting the time derivative of an integer order by Riemann-Liouville fractional calculus operators. This generalization agrees one to define precisely non-integer order integrals or derivatives. For describing viscoelastic behavior, fractional derivatives have been considerably flexible [4-6].

Anomalous diffusion is one of the most ubiquitous phenomena in nature [7], which has a large variety of physical situations. For instance, surface growth phenomena, transport of fluid in porous media [8], two-dimensional rotating flow [9], diffusion at liquid surfaces [10], diffusion of plasma [11], sub-recoil laser cooling [12], continuous-time 
random walks $[13,14]$ and non-Markovian dynamical processes in protein folding [15]. Anomalous diffusion deviates from the standard Fichean description of Brownian motion. Such anomalous behavior can be represented by Lévy processes whose main character is that the mean squared displacement is a nonlinear growth with respect to time, such as $\left\langle x^{2}>\sim t^{\gamma}\right.$. The time-fractional equation considered in this article describes the anomalous subdiffusion corresponding to $0<\gamma<1$. Sub-diffusion typically arises in cases where there are spatial or temporal constraints such as occur in porous media and fractal lattices.

Note that similar investigations have been made for the generalized second-grade fluid theoretically in terms of Wright function and Fox's $H$-function [16-18]. In this paper, the velocity and stress field of the generalized second-grade fluid with fractional anomalous diffusion caused by a plate moving impulsively on its own plate is investigated. The velocity and stress fields of the flow are explained by fractional-order differential equations. Exact analytic solutions of these differential equations are gained by applying the discrete Laplace transform technique of the sequential fractional derivatives method in terms of Fox's Hfunction.

\section{Problem Formulation}

The second-order approximation of the general constitutive equation given by Rivlin and Ericksen [19] can be written in the following form [20]

$$
T=-p I+\mu A_{1}+\alpha_{1} A_{2}+\alpha_{2} A_{1}^{2},
$$

where $T$ is the stress tensor, $-p$ is the pressure, $I$ is the unit vector, $\mu, \alpha_{1}$ and $\alpha_{2}$ are the constants of the fluid, customarily known as the coefficients of viscosity, viscoelasticity and cross-viscosity, respectively. $A_{1}$ and $A_{2}$ are the kinematical tensors defined through $[21,22]$

$$
\begin{gathered}
A_{1}=\nabla V+(\nabla V)^{T}, \\
A_{2}=\partial_{t} A_{1}+A_{1}(\nabla V)+(\nabla V)^{T} A_{1},
\end{gathered}
$$

where $\partial_{t}$ denotes the material time derivatives, $V$ denotes the velocity.

For fluid of the type (1) that is compatible with thermodynamics, in the sense that all the motions of the fluid encounter Clausius-Duhem inequality and the assumption that the Helmholtz free energy is minimum when the fluid is in equilibrium, the following constraints of the signs of the material moduli hold [23]

$$
\mu \geq 0, \alpha_{1} \geq 0, \alpha_{1}+\alpha_{2}=0
$$

Fluids meeting the above requirements are well-known as second-grade fluids in the literature. Generally, the constitutive equation of generalized second-grade fluids also has the form (1) but $A_{2}$ is defined as follows [24]

$$
A_{2}=\partial_{t}^{\beta} A_{1}+A_{1}(\nabla V)+(\nabla V)^{T} A_{1}
$$

where Caputo fractional calculus operator denoted by $\partial_{t}^{\beta}$ and may be defined as

$$
\partial_{t}^{\beta} f(t)=\frac{1}{\Gamma(1-\beta)} \int_{0}^{t}(t-\tau)^{-\beta} f^{\prime}(\tau) d \tau, \quad 0<\beta<1,
$$

where $\Gamma($.$) is the Gamma function, \partial_{t}^{\beta}$ represents the material time derivative of fractional order and $f^{\prime}$ denotes the ordinary derivative. When $\beta=0$, and $\alpha_{1}=0$, then the relationship illustrates the classical viscous Newtonian fluid.

In the exclusion of body force, the equation of motion is

$$
\rho \frac{D V}{D t}=\Delta \cdot T
$$

Where $\rho$ is the density, and $\frac{D}{D t}$ is the time fractional material derivative.

The equation of continuity for the velocity is

$$
\Delta . V=0 .
$$

We seek a solution for the velocity field in the form

$$
V=u(y, t) i \text {. }
$$

The fluid is set into motion through the action of the stress at the plate. The stress is described by (1), (2), (4) and (5). By substitution $u(y, t)$ in the equations, we obtain

$$
T_{x y}=\mu \partial_{y} u+\alpha_{1} \partial_{t}^{\beta} \partial_{y} u,
$$

where $T_{x x}=T_{y y}=T_{z z}=T_{x z}=T_{y z}=0$ and $T_{x y}=T_{y x}$.

By inserting the stress components and the velocity in (7), we obtain the modified Navier-Stokes equation

$$
\rho \partial_{t}^{\alpha} u=\mu \partial_{y}^{2} u+\alpha_{1} \partial_{t}^{\beta} \partial_{y}^{2} u
$$

where $\partial_{t}^{\alpha}$ and $\partial_{t}^{\beta}$ denote time-fractional derivative, and the fluid is generalized second-order fluid, respectively.

For the impulsive motion, the initial and boundary conditions are as follows:

$$
\begin{aligned}
& u(y, 0)=0 \text { for } y>0, u(0, t)=U \text { for } t>0, \\
& u \rightarrow 0 \text { for } \mathrm{y} \rightarrow \infty
\end{aligned}
$$

\section{Velocity Field}

In this study, dimensionless variables are introduced as follows: 
$u^{*}=\frac{u}{U}, y^{*}=\frac{y U}{v}, t^{*}=\frac{t U^{2}}{v}, \eta^{*}=\frac{\alpha_{1} U^{2}}{\rho v^{2}}$, where $v=\frac{\mu}{\rho}$.

$U$ and $\eta$ denote characteristic velocity and dimensionless parameter, respectively. Applying these dimensionless variables, governing equation of (11) and initial and boundary conditions (12) can be written as

$$
\begin{gathered}
\partial_{t}^{\alpha} u=\left(1+\eta \partial_{t}^{\beta}\right) \partial_{y}^{2} u \\
u(y, 0)=0 \text { for } y>0, u(0, t)=1 \text { for } t>0, \\
u \rightarrow 0 \text { for } \mathrm{y} \rightarrow \infty
\end{gathered}
$$

The asterisks symbol is omitted here for its simplicity. Let us assume that

$$
\bar{u}(y, s)=L\{u(y, t), s\}=\int_{0}^{\infty} e^{-s t} u(y, t) d t \text { is the image function }
$$

of $u(y, t)$, where $s$ is the parameter of transformation. In equation (13) and (14), we use the Laplace transform principle of sequential fractional derivatives,

$$
\frac{d^{2} \bar{u}}{d y^{2}}-\frac{s^{\alpha}}{1+\eta s^{\beta}}=0, \bar{u}(0, s)=\frac{1}{s}, \bar{u} \rightarrow 0 \text { for } y \rightarrow \infty, \quad \text { whose }
$$
analytic solution is

$$
\bar{u}(y, s)=\frac{1}{s} \exp \left\{-s^{\alpha / 2} y /\left(1+\eta s^{\beta}\right)^{1 / 2}\right\} .
$$

Now, we can rewrite (15) as follows

$$
\bar{u}(y, s)=\frac{1}{s}+\sum_{n=1}^{\infty} \frac{(-y)^{n} s^{n \alpha / 2-1}}{n !\left(1+\eta s^{\beta}\right)^{1 / 2}}=\frac{1}{s}+\sum_{n=1}^{\infty} \frac{\left(-\eta^{-1 / 2} y\right)^{n}}{n !} \sum_{m=0}^{\infty} \frac{\left(-\eta^{-1}\right)^{m} \Gamma(m+n / 2)}{m ! \Gamma(n / 2)} \frac{1}{s^{-n \alpha / 2+\beta n / 2+\beta m+1}} .
$$

Applying the discrete inverse Laplace transform into (16), we get

$$
\begin{gathered}
u(y, t)=1+\sum_{n=1}^{\infty} \frac{\left(-y \eta^{-1 / 2} t^{(\beta-\alpha) / 2}\right)^{n}}{n !} \sum_{m=0}^{\infty} \frac{\left(-\eta^{-1} t^{\beta}\right)^{m} \Gamma(m+n / 2)}{m ! \Gamma(n / 2) \Gamma(n(\beta-\alpha) / 2+\beta m+1)} \\
=1+\sum_{n=1}^{\infty} \frac{\left(-y \eta^{-1 / 2} t^{(\beta-\alpha) / 2}\right)^{n}}{n !} H_{13}^{11}\left[\left.\eta^{-1} t^{\beta}\right|_{(0,1) ;\left(1-\frac{n}{2}, 0\right) ;\left(\frac{-n(\beta-\alpha)}{2}, \beta\right)} ^{\left(1-\frac{n}{2}\right)}\right),
\end{gathered}
$$

in which $H_{p, q}^{m, n}(z)$ denotes Fox's H function [25]. Now let us make some analyses for the formula (17).

(i) When $\alpha=1, \beta=\alpha_{1}=0,(17)$ can be written as

$$
u(y, t)=1+\sum_{n=1}^{\infty} \frac{\left(-y \eta^{-1 / 2} t^{-1 / 2}\right)^{n}}{n ! \Gamma(1-n / 2)}\left(1+\eta^{-1}\right)^{-n / 2}=\sum_{n=0}^{\infty} \frac{\left(-y t^{-1 / 2}\right)^{n}}{n ! \Gamma(1-n / 2)}=W_{-1 / 2,1}\left(-y t^{-1 / 2}\right)
$$

in which $W_{p, q}(z)=\sum_{n=0}^{\infty} \frac{z^{n}}{\Gamma(p n+q)}, z \in \mathbb{C}$ is the Wright function [26]. Using the definition of the Wright function and the series expression of the error function, we can find the following relation [16]

$$
W_{-1 / 2,1}(z)=\operatorname{erfc}(-z / 2)
$$

Substituting (19) into (18), we have $u(y, t)=W_{-1 / 2,1}\left(-y t^{-1 / 2}\right)=\operatorname{erfc}(-y / 2 \sqrt{t})$.

This is the classical Rayleigh's analogy solution of Newtonian fluid [27]. It is evident that the result of this paper includes a particular case the classical Newtonian fluid.

(ii) When $\beta=1$, formula (17) can be simplified as

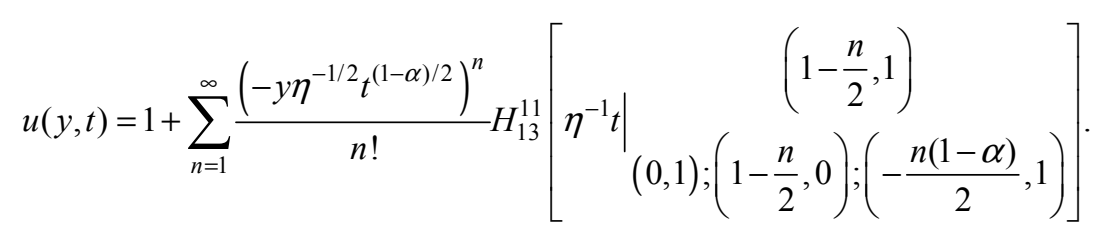


This is the solution for velocity for ordinary second-order fluid.

(iii) When $\beta=\alpha_{1}=0,0<\alpha<1$, (17) leads us,

$$
u(y, t)=\sum_{n=0}^{\infty} \frac{\left(-y t^{-\alpha / 2}\right)^{n}}{n ! \Gamma(1-n \alpha / 2)}=W_{-\alpha / 2,1}\left(-y t^{-\alpha / 2}\right) .
$$

Separating (20) into two parts according to the odd and even terms and applying formulas

$$
\Gamma(1 / 2-n)=(-1)^{n} 2^{n} \sqrt{\pi} /(2 n-1) ! ! \text { and } \Gamma(-1 / 2-n)=(-1)^{n+1} 2^{n+1} \sqrt{\pi} /(2 n+1) ! !
$$

We have

$$
u(y, t)=\pi^{-1} H_{23}^{21}\left[\begin{array}{c|c}
\frac{y t^{-\alpha / 2}}{2} & (1,1) ;\left(1, \frac{\alpha}{2}\right) \\
\left(\frac{1}{2}, \frac{1}{2}\right) ;\left(1, \frac{1}{2}\right) ;(0,1)
\end{array}\right],
$$

which is the solution of the fractional diffusion equation obtained by Wyss [28], using contour integral and inverse Mellin transform method to find the solution.

\section{Stress Field}

Since the fluid is set into motion through the action of the stress at the plate, the stress field ought to be computed. From (10) the dimensionless stress can be reprepresnted

$$
F=\frac{\partial u}{\partial y}+\eta D_{t}^{\beta} \frac{\partial u}{\partial y}
$$

where $F(y, t)=\frac{T_{x y}}{\rho U^{2}}$.

The Laplace transform of (22) is

$$
\begin{gathered}
\qquad \bar{F}(y, s)=\frac{d \bar{u}(y)}{d y}+\eta s^{\beta} \frac{d \bar{u}(y)}{d y} . \\
\text { Substituting (15) into (23), we have } \bar{F}(y, s)=-\frac{s^{\alpha / 2-1}}{\left(1+\eta s^{\beta}\right)^{-1 / 2}} \exp \left(-y\left(\frac{s^{\alpha}}{1+\eta s^{\beta}}\right)^{1 / 2}\right) .
\end{gathered}
$$

We apply the discrete inverse Laplace transform formula again to give the stress distribution. Firstly, we rewrite (24) as a series form

$$
\bar{F}(y, s)=-\sum_{k=0}^{\infty} \frac{(-y)^{k} s^{(k+1) \alpha / 2-1}}{k !\left(1+\eta s^{\beta}\right)^{(k-1) 1 / 2}}=-\sum_{k=0}^{\infty} \frac{(-y)^{k}\left(\eta^{-1}\right)^{\frac{k-1}{2}}}{k !} \sum_{n=0}^{\infty} \frac{\left(-\eta^{-1}\right)^{n} \Gamma\left(\frac{k-1}{2}+n\right)}{n ! \Gamma\left(\frac{k-1}{2}\right)} \frac{1}{s^{-(k+1) \alpha / 2+\beta n+(k-1) \beta / 2+1}} .
$$

Using the discrete inverse Laplace transform with (25), we obtain

$$
F(y, t)=-\sum_{k=0}^{\infty} \frac{(-y)^{k}\left(\eta^{-1}\right)^{\frac{k-1}{2}}}{k !} \sum_{n=0}^{\infty} \frac{\left(-\eta^{-1} t^{\beta}\right)^{n} \Gamma\left(\frac{k-1}{2}+n\right)}{n ! \Gamma\left(\frac{k-1}{2}\right)} \frac{t^{-(k+1) \alpha / 2+(k-1) \beta / 2}}{\Gamma\left(-\frac{k+1}{2} \alpha+\beta n+\frac{k-1}{2} \beta\right)}
$$




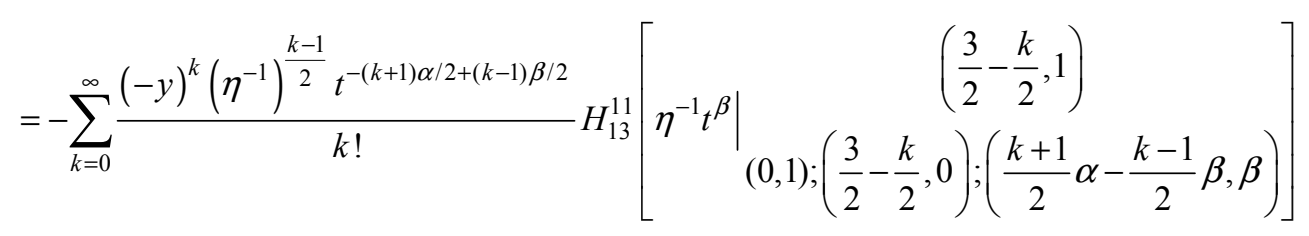

Now let us make some analyses for the above formulas of this study

(i) If $\beta=\alpha_{1}=0, \alpha=1$, the medium is a Newtonian fluid, and (26) becomes

$$
F(y, t)=-t^{-1 / 2} \sum_{k=0}^{\infty} \frac{\left(-y t^{-1 / 2}\right)^{k}}{k ! \Gamma\left(\frac{1}{2}-\frac{k}{2}\right)}=-t^{-1 / 2} W_{-1 / 2,1 / 2}\left(-y t^{-1 / 2}\right) .
$$

By the definition of Wright function, we are able to demonstrate that

$$
W_{-1 / 2,1 / 2}(-z)=\pi^{-1 / 2} \exp \left(-z^{2} / 2\right) .
$$

Substituting (28) into (27), we can reduce (27) to the classical solution of Newtonian fluid.

(ii) If $\alpha=1$, then (26) can be simplified as

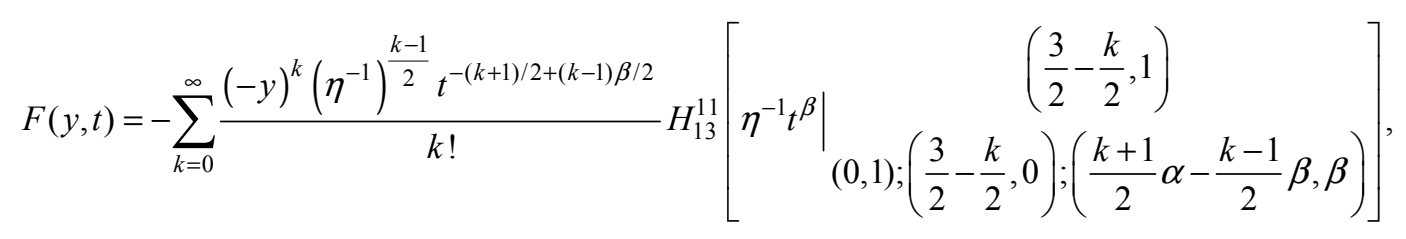

which is the same result as shown by Tan and $\mathrm{Xu}$ [16].

To obtain the value of the shear stress at the plate, we put $y=0$ in (24), to get the following expression

$$
\bar{F}_{p}(s)=\frac{-s^{\alpha / 2-1}}{\left(1+\eta s^{\beta}\right)^{-1 / 2}} .
$$

Similarly, we obtain the following formula to calculate the shear stress at the plate

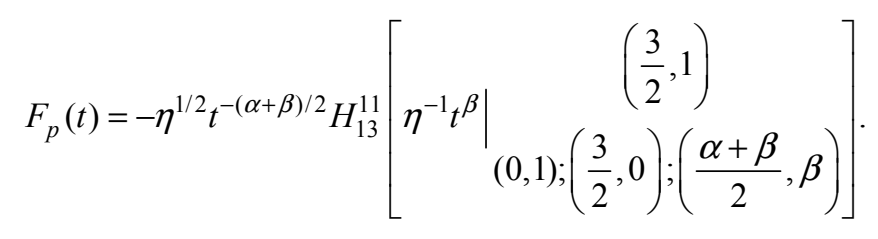

\section{The Relationship Between Stress Field and Velocity Field}

Substituting (15) into (24), we obtain

$$
\bar{F}(y, s)=-s^{\alpha / 2}\left(1+\eta s^{\beta}\right)^{1 / 2} \cdot \bar{u}(y, s) .
$$

Similarly, we can obtain the Laplace inversion of the first term at the right-hand side of the equation

$g(t)=L^{-1}\left\{-s^{\alpha / 2}\left(1+\eta s^{\beta}\right)^{1 / 2}, t\right\}=L^{-1}\left\{\eta^{1 / 2} \sum_{n=0}^{\infty} \frac{\left(-\eta^{-1}\right)^{n} \Gamma\left(n-\frac{1}{2}\right)}{n ! \Gamma\left(-\frac{1}{2}\right)} \frac{1}{s^{\beta n-(\alpha+\beta) / 2}}, t\right\}=\eta^{1 / 2} \sum_{n=0}^{\infty} \frac{\left(-\eta^{-1}\right)^{n} \Gamma\left(n-\frac{1}{2}\right)}{n ! \Gamma\left(-\frac{1}{2}\right)} \frac{t^{\beta n-((\alpha+\beta) / 2)-1}}{\Gamma\left(\beta n-\frac{\alpha+\beta}{2}\right)}$

Applying the convolution theorem of the Laplace transform with equation (31), we get 


$$
F(y, t)=-\eta^{1 / 2} \sum_{n=0}^{\infty} \frac{\left(-\eta^{-1}\right)^{n} \Gamma\left(n-\frac{1}{2}\right)}{n ! \Gamma\left(-\frac{1}{2}\right) \Gamma\left(\beta n-\frac{\alpha+\beta}{2}\right)} \int_{0}^{t}(t-\tau)^{\beta n-((\alpha+\beta) / 2)-1} u(y, \tau) d \tau .
$$

Using the definition of fractional calculus, equation (32) can be written as

$$
F(y, t)=-\eta^{1 / 2} \sum_{n=0}^{\infty} \frac{\left(-\eta^{-1}\right)^{n} \Gamma\left(n-\frac{1}{2}\right)}{n ! \Gamma\left(-\frac{1}{2}\right)} \cdot D_{t}^{-\beta n+(\alpha+\beta) / 2} u(y, t) .
$$

The physical meaning of (32) and (33) is that the stress at a given point at any time is depended on the time history can be depicted by the fractional calculus. If one puts $\alpha=1, \beta=$ $\alpha_{1}=0$, formula (33) can be simplified as $f(y, t)=D_{t}^{1 / 2} u(y, t)$. This is the dimensionless form of the result obtained by

Bagley and Torvic [29].

\section{Conclusions}

In this study, the classical problem of the plate moving impulsively in its own plane to the generalized second-order fluid is extended by replacing material time derivative of integer order with fractional-order and defining $0<\beta \leq 1$. Some important results presented for the flow field of a generalized second-grade fluid with anomalous sub-diffusion $(0<\alpha \leq 1)$ near the wall suddenly set in motion. The discrete Laplace transform method of sequential fractional derivatives is used to obtain exact analytical solutions of velocity and stress. The fractional derivatives approach in the constitutive relationship model of generalized viscoelastic fluid and anomalous diffusion are introduced. The fractionalorder model is more applicable than the ordinary second-order model narrating the properties of viscoelastic fluid. We hope that our model and the analytical solutions employed in this paper will be used to validate and interpret more complicated types of flow and the analysis of viscoelastic fluid.

\section{References}

[1] T. Hayat, A. M. Siddiqu and S. Asghar. Some simple flows of an Oldroyd-B fluid. Int. J. Eng. Sci., 39: 135-147, 2001.

[2] W. C. Tan and M. Y. Xu. Unsteady flows of a generalized second grade fluid with the fractional derivative model between two parallel plates. Acta. Mech. Sin., 20 (5): 471-476, 2004.

[3] T. Hayat, Naveed Ahmed, M. Sajid and S. Asghar. On the MHD flow of a second grade fluid in a porous channel. Comput. and Math. with Appl., 54: 407-414, 2007.

[4] H. Schiessel, R. Metzler, A. Blumen and T. F. Nonnemacher. Generalized viscoelastic models: their fractional equations with solutions. J. Phys. A: Math. Gen., 28: 6567-6584, 1995.

[5] L. I. Palade, P. Attane, R. R. Huilgol and B. Mena. Anomalous stability behavior of a properly invariant constitutive equation which generalises fractional derivative models. Int. J. Eng. Sci., 37: 315-329, 1999.

[6] Y. A. Rossihin and M. V. Shitikova. A new method for solving dynamic problems of fractional derivative viscoelasticity. Int. J. Eng. Sci., 39: 149-176, 2001.

[7] J. P. Bouchaud and A. Georges. Anomalous diffusion in disordered media: Statistical mechanisms, models and physical applications. Phys. Rep., 195: 127-293, 1990.

[8] H. Spohn. Surface dynamics below the roughening transition. J. Phys., 3 (1): 69-81, 1993.

[9] T. H. Solomon, E. R. Weeks and H. L. Swinney. Observation of anomalous diffusion and Lévy flights in a two-dimensional rotating flow. Phys. Rev. Lett., 71: 3975-3978, 1993.

[10] J. Buckmaster. Viscous sheets advancing over dry beds. $J$. Fluid Mech., 81: 735-756, 1977.

[11] J. R. Drake and J. G. Berryman. Theory of nonlinear diffusion of plasma across the magnetic field of a toroidal multipole. Phys. Fluids, 20 (5): 851-857, 1977.

[12] F. Bardou, J. P. Bouchaud, O. Emile. A. Aspect and C. CohenTannoudji. Subrecoil laser cooling and Lévy flights. Phys. Rev. Lett., 72: 203-206, 1994.

[13] R. Hilfer. Applications of Fractional Calculus in Physics. World Scientific, Singapore, 2000.

[14] R. Metzler and J. Klafter. The random walk's guide to anomalous diffusion: A fractional dynamics approach. Phys. Rep., 339: 1-77, 2000.

[15] S. S. Plotkin and P. G. Wolynes. Non-Markovian configurational diffusion and reaction coordinates for protein folding. Phys. Rev. Lett., 80: 5015-5018, 1998.

[16] W. C. Tan and M. Y. Xu. The impulsive motion of flat plate in a generalized second grade fluid. Mech. Res. Commun., 29: 3$9,2002$.

[17] Moustafa El-Shahed. On the impulsive motion of flat plate in a generalized second grade fluid. Z. Naturforsch., 59a: 829837, 2004.

[18] A. Khan, G. Zaman and O. Algahtani. Unsteady flow of viscoelastic fluid due to impulsive motion of plate. Asian journal of mathematics and applications, Article Id. ama0191, 9 pages, 2014.

[19] R. S. Rivlin and J. L. Ericksen. Stress deformation relations for isotropic materials. J. Rational Mech. Anal., 4: 323-425, 1955. 
[20] A. M. Siddiqui, T. Hayat and S. Asghar. Periodic flows of a non-Newtonian fluid between two parallel plates. Int. J. NonLinear Mech., 34: 895-899, 1999.

[21] N. C. Ghosh, B. C. Ghosh and L. Debnath. The Hydromagnetic flow of a dusty viscoelastic fluid between two infinite parallel plates. Comput. Math. Appl., 39: 103-116, 2000 .

[22] P. D. Ariel. On exact solutions of flow problems of a second grade fluid through two parallel porous walls. Int. J. Eng. Sci., 40: 913-941, 2002.

[23] R. L. Fosdick and K. R. Rajagopal. Anomalous features in the model of second order fluids. Arch. Ration. Mech. Anal., 70: 145-152, 1979.

[24] A. Mahmood, C. Fetecau, N. A. Khan and M. Jamil. Some exact solutions of the oscillatory motion of a generalized second grade fluid in an annular region of two cylinders. Acta. Mech. Sin., 26: 541-550, 2010.

[25] A. M. Mathai and R. K. Saxena. The H-function with applications in statistics and other disciplines. New DelhiBangalore-Bombay: Wiley Eastern Limited, 1978.

[26] R. Gorenflo, Y. Luchkoa and F. Mainardi. Wright functions as scale-invariant solutions of the diffusion-wave equation. $J$. Comput. Appl. Math., 118 (1): 175-191, 2000.

[27] G. K. Batchelor. An Introduction to Fluid Dynamics. Cambridge University press, Cambridge, 1967.

[28] W. Wyss. The fractional diffusion equation. J. Math. Phys., 27 (11): 2782-2785, 1986.

[29] R. L. Bagley and P. J. Torvik. On the appearence of fractional derivative in the behavior of real materials. J. Appl. Mech. 51, 294, 1984. 